\title{
ASPECTS OF ULTRAFAST SPARK GAP SWITCHING FOR UWB HPM GENERATION
}

\author{
J.M. Lehr, C.E. Baum, W.D. Prather and F.J. Agee \\ High Energy Sources Division, Phillips Laboratory \\ Kirtland AFB, New Mexico 87117
}

\begin{abstract}
The Air Force is interested in cornpact ultra-wideband systems which utilize a minimum volume of high pressure gas. These desires lead us to look closely at single channel spark gaps, because both the size and volume of gas, for example, hydrogen, under pressure can be much less than needed for sources containing ring gap switches. While single channel spark gap switches are desirable, the intrinsic inductance of the spark gap is prohibitively high to achieve large rates of voltage rise.

For future applications, the limit of spark gap technology for ultrafast switching is explored. Of primary interest is the fastest possible risetime achievable with a single channel spark gap. Thus far we have calculated the limit on the achievable risetime with spark gap technology, using three different approaches, which are all in good agreement. The first examines the excitation rates in gases to determine its limitations. The second assumes a streamer mechanism and uses the velocity of propagation to estimate the achievable risetime. The third utilizes an equivalent circuit model.

It is commonly believed that the impedance mismatch in the spark region, caused by the additional spark gap inductance, is unavoidable. To reduce the effect of the intrinsic inductance of the channel, the High Energy Source Division has devised a simple geometrical alteration to the spark gap geometry which reduced the inductance per unit length of the spark gap to that of its transmission line feed. This is anticipated to permit the realization of picosecond risetime UWB HPM sources.
\end{abstract}

\section{INTRODUCTION}

The general limitation on the achievable risetime for gaseous ultra-wideband (UWB) high power microwave (HPM) sources is the spark gap. It is of interest, for a myriad of applications, to produce, control and accurately measure ultrafast breakdown in spark gaps. Thus, knowledge of the fundamental limitations of spark gap technology is desirable. Three treatments are used to examine the limits. The first examines the rate of carrier generation in the gaseous insulating media under uniform field conditions to estimate the minimum time to achieve the critical charge carrier density. Secondly, the limitation imposed by the finiteness of the electromagnetic propagation. Finally, a circuit model for the post-breakdown circuit is invoked to derive the limitation on the rate of voltage rise. These treatments provide insight into ultrafast breakdown and guidelines for its generation. 


\section{Peaking Spark Gaps}

Pulse conditioning systems are being used to generate UWB High Power Microwave (HPM) in the 10 's of gigawatt power range. [1,2] In general, this is accomplished by successively manipulating the timescale at which energy is delivered to a load. A block diagram of such a power conditioning system, is shown in Figure 1. Pulsewidths in the sub-nanosecond regime are produced by charging transmission lines of successively shorter electrical length along with an accompanying increase in voltage.

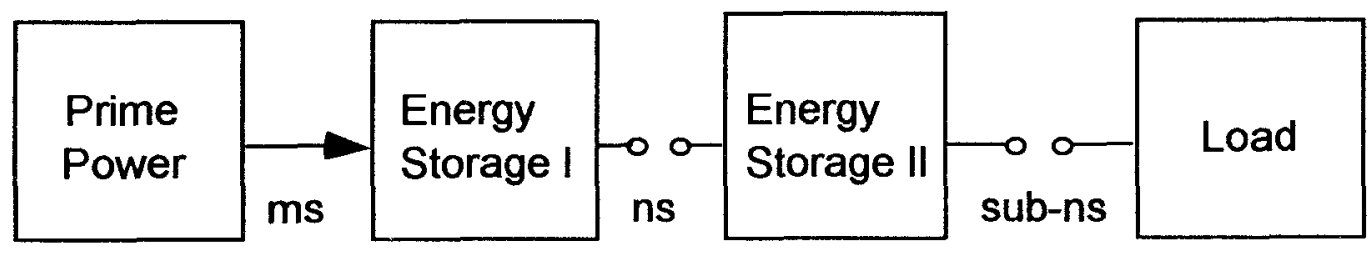

\section{Kilowatt $\longrightarrow$ Megawatt $\longrightarrow$ Gigawatt}

Figure 1. UWB HPM is produced by radiating high power pulses of very short duration. Subnanosecond duration pulsed are generated by successively reducing the timescale at which electrical energy is stored.

The switching element is a major component of any power conditioning system and, for UWB HPM generation, ultrafast closing capability, along with fast voltage recovery are desired. A fast pulse risetime is critical because the risetime contains the high frequency components of the resulting spectrum. To sharpen the risetime on a pulse, a spark gap configuration, called a peaking gap is used. The crux of the peaking gap is the establishment of very high electric fields in the interelectrode spacing. The velocity of propagation of the electron avalanche is proportional to the electric field applied to electrodes, and thus, gap closure is dominated by the applied electric field. To produce ultrafast switching, the spark gap is dramatically overvolted; that is, the spark gap is charged far in excess of its self-breakdown voltage. Peaking gaps typically operate at gas pressures in the range of $100 \mathrm{~atm}$ and electric fields in the $\mathrm{MV} / \mathrm{cm}$ range. The self breakdown curve for gases is known to saturate in the vicinity of $100 \mathrm{MV} / \mathrm{m}$ for pressures to $50 \mathrm{~atm}$.[3] To achieve overvolting without switching at the self breakdown voltage, the spark gap is pulse charged very quickly. This allows a large overvoltage to be achieved, and overvoltages of over $300 \%$ are achievable.

Interelectrode gap distance, $\delta$, is chosen as small as possible to minimize the intrinsic inductance of the spark channel, since the inductance is known to limit the achievable risetime of the resultant pulse. Spark channel inductances of less than $1 \mathrm{nH}$ have been achieved with gap lengths of $1 \mathrm{~mm}$ and less. Because of the short gap lengths, even charge voltages of less than $100 \mathrm{kV}$ can produce interelectrode electric fields in the $\mathrm{MV} / \mathrm{cm}$ range with voltage doubling at the spark gap. These small interelectrode distances, however, yield high spark gap capacitances, even for relatively small diameter electrodes. Moreover, this high spark gap capacitance, and the fast charging times lead to 
a strong displacement current which manifests as an undesirable prepulse on the load voltage. The prepulse is observed on the output pulse with a magnitude, $V_{p p}$, which is related to the capacitance of the spark gap, C, by

$$
V_{p p}=Z_{0} C \frac{d V_{C}}{d t}
$$

where $Z_{0}$ is the output impedance and $V_{C}$ is the voltage which is applied to the spark gap. Since a fast charge is critical to peaking gap operation, small diameter electrodes are desirable. Moreover, minimizing the electrode diameter of the peaking gap may lead to enhanced performance. The generation of $50 \mathrm{ps}$ risetime pulse with a $60 \mathrm{kV}$ charge in a single channel switch of very small dimensions has been reported. [4]

\section{PREDICTIVE RELATIONS}

The spark gap is said to have closed when the potential difference between the electrodes is sharply reduced and current becomes circuit limited. The predictions for the limitation of spark gap technology are broken into two parts: the prebreakdown limitation and the post breakdown limitation. The development of the prebreakdown phase of the spark development determines the minimum closure time of the spark gap, which determines the risetime. Once the voltage across the channel has collapsed, the electromagnetic wave must re-establish in the interradial distance. The post breakdown phase consists of the inhibition of the voltage rise due to the inductance of the spark channel itself.

\section{Avalanche Propagation Limit}

The breakdown of a spark gap initiates with an electron avalanche, even when a streamer mechanism is operative. An avalanche initiates from an initial electron distribution which may be resident in the gas, or from an external source. If the initial electrons are thermally distributed, the electron population is small. The electrons accelerate under the influence of an external field. After some time, the electron undergoes a collision with the background gas, with a resultant energy exchange. If this energy exchange is sufficient, an ionizing collision occurs and an electron/ion pair is created. The growth of the current density in this manner results in an electron avalanche. The growth of the electron avalanche is described by the evolution of the electron number density. The electron number density, $N(t)$, grows from its initial value, $N_{0}$, under the influence of an applied electric field, according to

$$
N(t)=N_{0} \exp ^{\int \alpha(v) v d t}
$$

where $\mathrm{v}$ is the velocity of the electron, $\mathrm{t}$ is time, and $\alpha(\mathrm{v})$ is the electron ionization rate coefficient. In general, the rate coefficient, $\alpha$, is generally a function of the relative velocity of the collision pair and is gas specific. The above equation leads to the definition of the time constant related to the 
above equation, the avalanche growth time, given by

$$
t_{a}=\frac{1}{\alpha \nu} \text {. }
$$

For gas pressures of $100 \mathrm{~atm}$, avalanche transit times on the order of 1 ps has been calculated for reduced electric field, $\mathrm{E} / \mathrm{p}$ of $90 \mathrm{~V} / \mathrm{cm}$-torr.[5] If a single avalanche results in breakdown, the associated risetime for avalanche growth is the risetime of the pulse. This treatment however assumes the absence of the streamer mechanism and, thus, inherently assumes uniform field conditions apply.

It should be noted that this estimation is still valid when a streamer mechanism is operative. The streamer mechanism initiates with an electron avalanche. The electron avalanche grows until the electric field produced by the cumulative space charge in the avalanche head is on the order of the applied field. The critical electron number for the avalanche to streamer transition is on the order of $10^{8}$. When this criteria is met, the discharge is said to have entered the streamer phase. The advent of the streamer mechanism serves to greatly enhance the propagation speed of the discharge. Thus, the major component of the discharge transit time is the electron avalanche development time.

\section{Electromagnetic Propagation Limit}

The electromagnetic propagation limit explores the timescale of the propagating wave in the insulating media. Prior to breakdown, a spark gap is a capacitor. This capacitor, however, does not necessarily charge fully before breakdown occurs. The spark gap cannot close in less time than the transit time of the electromagnetic wave though the gap, as shown in Figure 2.

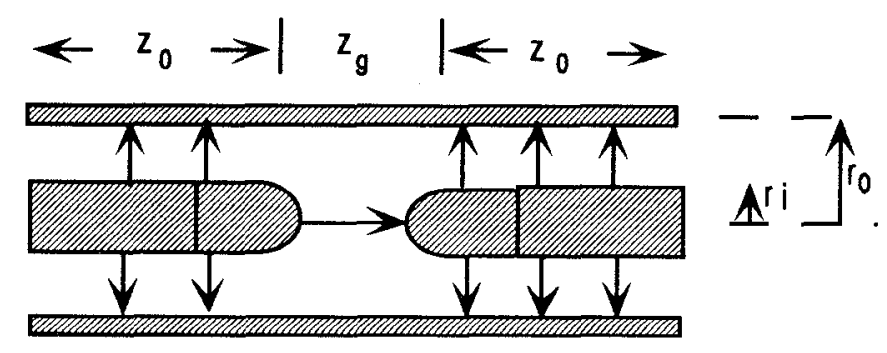

Figure 2. The electric field lines as a pulse propagates through a spark gap prior to breakdown. The field lines on the right hand side are the transmitted wave after the spark gap switches.

To examine the propagation time in the spark gap spacing, the common scaling parameter $\mathbf{P} \delta$ is used, where $\mathbf{P}$ is the gas pressure, and $\delta$ is the interelectrode gap distance. Thus, the transit time of an 
electromagnetic pulse through a spark gap filled with an insulating media with electric permittivity, $\epsilon$, and magnetic permeability, $\mu$, is

$$
P \delta=\frac{P t_{r}}{\sqrt{\epsilon \mu}}
$$

Thus for a high pressure gaseous spark gap, with an interelectrode gap spacing of $1 \mathrm{~mm}$, the minimum risetime is approximately $3 \mathrm{ps}$. However, for a spark gap inserted into a coaxial transmission line, the risetime is determined not only by the gap closure but also by the reestablishment of the propagating electric field in the inter-radial distance. The achievable risetime is longer since the electromagnetic wave must be re-established between the inner and outer conductors. Thus, the above limit must be modified by the interradial transit time, yielding,

$$
P[\delta+\Delta r]=\frac{P t_{r}}{\sqrt{\epsilon \mu}},
$$

where $\Delta r$ is the difference between the outer and inner radii of the transmission line. The resolution time, then, for measurements is given by $\Delta r(\epsilon \mu)^{1 / 2}$. For most high power systems, the interradial distance, $\Delta r$, is made large to insure voltage holdoff in the coaxial systems. Of course, the breakdown voltage is inversely proportional to both the inner radius and the logarithm of the radius ratio. Thus, for ultrafast switching, for a given impedance, the inner conductor radius should be chosen to be small.

\section{Maximum Rate of Voltage Rise}

Spark gaps are very good candidates for transferring high peak powers. However, the small radius of the spark channel makes these switches intrinsically inductive and limits the achievable risetime by inhibiting the rate of voltage rise. The spark gap is represented by the circuit of Figure 3, which is valid once the discharge channel current is circuit limited. From this simple equivalent circuit, the maximum rate of voltage rise, $\mathrm{dV} / \mathrm{dt}$, in the spark gap is calculated.

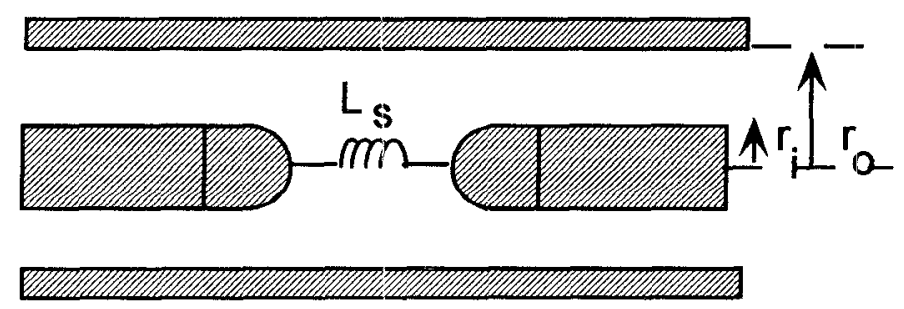

Figure 3. The equivalent circuit of the discharge channel of a spark gap. 
When breakdown has progressed so that it is self sustaining, the single channel of the spark gap is modeled as an inductor, $\mathrm{L}_{\mathrm{S}}$. The coaxial transmission line feed of the equivalent circuit has an inductance per unit length, $L^{\prime}$, given by

$$
L^{\prime}=\frac{\mu_{0}}{2 \pi} \ln \left[\frac{r_{o}}{r_{i}}\right],
$$

where $r_{o}$ and $r_{i}$ are the outer and inner radii of the transmission line. The value of the inductance of the spark channel, $\mathrm{L}_{\mathrm{S}}$, can be difficult to calculate exactly. However, it has been shown that a very good approximation can be obtained by treating the current carrying channel as a transmission line of inner radius $r_{c}$ and outer radius $r_{o}$.[6] Thus, the intrinsic inductance, $L_{s}$, of the current carrying breakdown channel is

$$
L_{S}=\delta \frac{\mu_{0}}{2 \pi} \ln \left[\frac{r_{o}}{r}\right]
$$

where $\delta$ is the interelectrode spacing of the spark gap. The inductance per unit length of the spark gap, $\mathrm{L}_{\mathrm{s}}^{\prime}$, is

$$
L_{S}^{\prime}=\frac{L_{S}}{\delta}
$$

For a system with electrodes which are large compared to the channel radius, the value of the inductance per unit length, $\mathrm{L}_{\mathrm{S}}{ }^{\prime}$, of the spark channel can be approximated. For a system with large electrodes, and reasonably low impedance,

$$
\frac{r_{o}}{r_{c}} \sim 30,50,100
$$

Since the natural logarithm function is slowly varying,

$$
\ln \left[\frac{r_{o}}{r_{i}}\right] \sim 4 \text {. }
$$

Thus, the inductance of the spark channel can be approximated for practical purposes as

$$
L_{S}^{\prime} \sim \frac{2 \mu_{0}}{\pi} .
$$

The voltage across this equivalent inductor of the spark channel is given by

$$
V=L \frac{d I}{d t}
$$

The voltage rate of rise is expressed as

$$
\frac{d V}{d t}=Z_{0} \frac{d I}{d t}
$$


where $Z_{0}$ is the feed impedance. Rearranging terms,

$$
\frac{d V}{d t}=\frac{Z_{0} V}{L_{S}} \text {. }
$$

If the electrodes of the spark gap are large, and spaced a distance, $\delta$, apart, the electric field is uniform and given by

$$
E=\frac{V}{\delta}
$$

The rate of voltage rise is related to the electric field by

$$
\frac{d V}{d t}=\frac{Z_{0} E}{L_{S}^{\prime}} \text {. }
$$

To estimate the maximum rate of voltage rise, let the electric field, $\mathrm{E}$, be $100 \mathrm{MV} / \mathrm{m}$, and the impedance be $50 \Omega$. Thus, the maximum rate of voltage rise is

$$
\frac{d V}{d t}=6 \cdot 10^{15} \frac{V}{s}
$$

This estimate implies that for a switching voltage of $100 \mathrm{kV}$, the achievable risetime is on the order of $10 \mathrm{ps}$. However, for a switching voltage of $1 \mathrm{MV}, 100 \mathrm{ps}$ is possible.

This estimate of the maximum rate of voltage rise in a spark gap is the order of magnitude which is currently being produced through the use of peaking gaps.[1,4] The above equation suggests that the even higher rates of voltage rise can be achieved by increasing the electric field on the spark gap and reducing the inductance per unit length of the spark channel.

\section{The Compensated Spark Gap: A Minimum Inductance Design}

In ultrafast switching, the interelectrode spacing in the spark gap is chosen to be very short to minimize the inductance of the spark channel. It can be shown, in general, the inductance of the spark channel in a spark gap dominates switch performance. This implies that by removing the additional inductance caused by the reduced crossectional area of the spark channel in relation to the electrode area, the switch characteristics will be completely determined by the inductance of the switch hardware. For long gap spark channels this can be accomplished by the addition of a "taper" in the outer conductor as shown in Figure 4.

The purpose of this taper is to match the impedance of the spark channel to the driving system impedance and hence, matches the inductance per unit length of the hardware to the inductance per unit length of the interelectrode gap region. As shown in Figure 4, the sharp edges introduce additional field enhancement to the spark gap design. A practical design contours both the inner 
conductor and the outer conductor to maintain a constant impedance as well as the voltage holdoff throughout the switch.

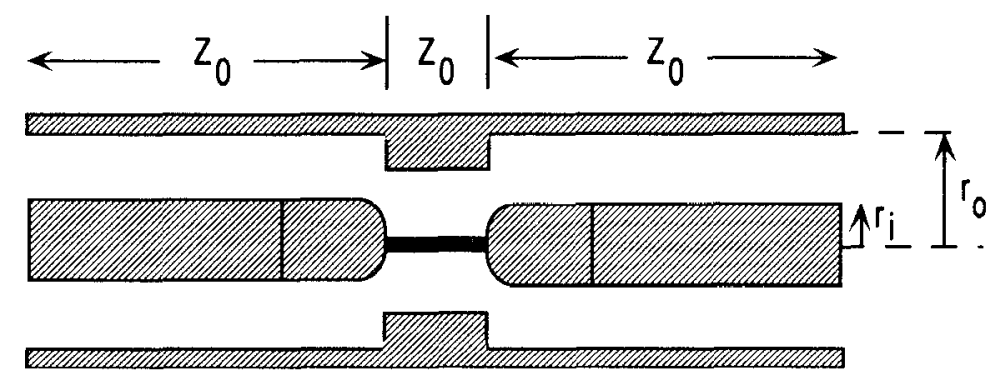

Figure 4. The added dimension of the outer conductor compensates for the reduced impedance of the spark channel radius. This technique matches the impedance of the spark channel to the driving system impedance. The taper dimension is chosen so that the ratio $r_{o} / r_{c}$ is equal to the ratio $r_{o} / r_{a}$.

Thus, fast single channel spark gap switching is optimized when the channel inductance per unit length is that of the transmission line feed. Additionally, the system impedance is maintained throughout the switch region, which eliminates the associated power loss. Thus, the notched spark gap design provides the fastest risetime, while maintaining the system impedance mismatch.

\section{CONCLUSIONS}

The peak risetime which can be achieved has been examined in three ways: investigation of the critical carrier production rate to produce an electron avalanche which results in closure of a gaseous spark gap; an examination of the limits imposed by the propagation of an electromagnetic wave in a transmission line encased spark gap; the third method results from a circuit model of the spark gap after breakdown. These calculations are in remarkably good agreement: risetimes on the order of 1-10 ps can be expected. Moreover, the calculations are independent, and thus, are not exclusive, but complementary.

The above calculations provide guidelines for peaking switch design. Foremost, the electric field which is applied to the switch is critical in determining the achievable risetime. The electromagnetic wave calculations indicate the inner conductor dimensions should be minimized for a given impedance. It has been shown that different expectations are valid for achieving peak voltages of different magnitudes. Moreover, the notched spark gap design may alleviate the conclusions regarding the maximum rate of rise. 


\section{REFERENCES}

[1] W.D. Prather, C.E. Baum, F.J. Agee, J.P. O'Laughlin, D.W. Scholfield, J.W. Burger, J.Hull, J.S.H. Schoenberg, and R. Copeland, "Ultrawide Band Sources and Antennas: Present Technology, Limitations, Future Challenges," Ultra Wideband/Short Pulse

Electromagnetics 3, Plenum Press, New York, 1996.

[2] J.P. OLaughlin and R.P. Copeland, "Subnanosecond Power Conditioning Technique Using Transmission Line to Transmission Line Charging," Proc. 20th Power Modulator Symposium, pp. 351-354, 1992.

[3] E.A. Avilov and N.V. Belkin, "Electrical Strength of Nitrogen and Hydrogen at High Pressures," Sov. Phys. Tech. Phys., Vol. 19, No. 12, June 1975.

[4] C.A. Frost, T.H. Martin, P.E. Patterson, L.F. Rinehart, G.J. Rohwein, L.D. Roose, J.F.Aurand, M.T.Buttram, "Ultrafast Gas Switching Experiments," Proc. 9th IEEE Pulsed Power Conference, K. Prestwich and W. Baker, Eds., pp. 491-494, 1993.

[5] R.E. Cassell and F. Villa,"High Speed Switching in Gases," SLAC Publication 1858, February, 1989.

[6] S. Levinson, E.E. Kunhardt and M. Kristiansen, "Simulation of Inductive and Electromagnetic Effects Associated with Single and Multichannel Triggered Spark Gaps," Proc. 2nd IEEE Pulsed Power Conference, A.H. Guenther and M. Kristiansen, Eds., pp. 433-436, 1979. 